\title{
Impacto en la calidad de vida de los adolescentes con diabetes mellitus tipo 1
}

\section{Impact on the quality of life of adolescents with diabetes mellitus type 1}

\author{
Francisca Lizama Fuentes ${ }^{\mathrm{a}}$, Sergio Ormeño Rojas ${ }^{\mathrm{a}}$, Francisca Mourguiart Liberona ${ }^{\mathrm{a}}$, \\ Joaquín Fuentes Cammella , Fanny López-Alegríab
}

aAlumno/a $5^{\circ}$ año de Enfermería, Facultad de Enfermería, Universidad Andrés Bello. Santiago, Chile

'Matrona, Facultad de Enfermería, Universidad Andrés Bello. Santiago, Chile

Recibido: 4 de mayo de 2020; Aceptado: 20 de julio de 2020

¿Qué se sabe del tema que trata este estudio?

Existen estudios que evidencian las alteraciones que ocasiona la diabetes mellitus tipo 1 en la salud física de los adolescentes, pero existe una escasez de estudios sobre el impacto de esta patología crónica en la calidad de vida de este grupo etario.
¿Qué aporta este estudio a lo ya conocido?

Este estudio aporta antecedentes sobre el impacto bidireccional de los niveles de hemoglobina glicosilada en la calidad de vida del adolescente que padece diabetes mellitus Tipo 1 .

\section{Resumen}

Introducción: La diabetes mellitus tipo 1 (DM1) es una enfermedad que se perfila para toda la vida. Objetivo: Identificar evidencia científica sobre el impacto de la DM1 en la calidad de vida de los adolescentes portadores de esta enfermedad. Método: Revisión sistemática en las bases bibliografías MEDLINE, LILACS, CINAHL y ScIELO, utilizando los descriptores "Adolescent*", "Teen*", "Diabetes Mellitus, Type1", "Diabetes, type 1", "Type 1 diabetes", "Quality of life", "Health related quality of life", "Life quality", "Health impact assessment", "Health impact", "Impact assessment, health", "Diabetes Impact Measurement Scales", "PedsQL", "Glycated Hemoglobin A1c", "Glycosylated Hemoglobin Alc", y "HbA1c". De los 679 artículos localizados, 25 fueron incluidos en el análisis. Algunos estudios fueron multicéntricos nacionales e internacionales. Los instrumentos más utilizados, relativos a las mediciones de la calidad de vida, fueron el Cuestionario de Calidad de Vida Pediátrica (PedsQL) en su versión genérica y módulo diabetes. Resultados: La calidad de vida evaluada por el adolescente que padece DM1 mediante Escalas de calidad de vida, está significativamente e inversamente asociada a los valores de HbAlc. Esta vinculación se extiende a una correlación significativa entre los puntajes genéricos totales de calidad de vida y $\mathrm{HbAlc}$, pero no tiene la misma repercusión en los puntajes específicos. Conclusiones: El control metabólico se establece como la piedra angular que incide en el impacto en la relación DM1 y calidad de vida; vinculación que se vislumbra como bidireccional, aunque no se evidencia un consenso absoluto sobre los tipos de factores y los grados que influirían en el control metabólico.
Palabras clave:

Adolescente;

Diabetes Mellitus

Tipo 1;

Calidad de Vida;

Evaluación del Impacto

en la Salud;

Hemoglobina A

Glicosilada 


\begin{abstract}
Introduction: Type 1 diabetes mellitus (DM1) is a chronic disease. Objective: To identify scientific evidence on the impact of DM1 on the quality of life of adolescents with this disease. Method: Systematic review in the bibliographic databases MEDLINE, LILACS, CINAHL, and ScIELO, using the following descriptors: "Adolescent *”, “Teen *”, "Diabetes Mellitus, Type1", "Diabetes, type 1", "Type 1 diabetes", "Quality of life", "Health related quality of life", "Life quality", "Health impact assessment”, "Health impact”, "Impact assessment, health", "Diabetes Impact Measurement Scales", "PedsQL", "Glycated Hemoglobin A1c", "Glycosylated Hemoglobin A1c", and "HbA1c". Out of 679 articles identified, 25 were included in the analysis. Some studies were national and international multicenter. The most widely used instruments related to quality of life measurements were the Pediatric Quality of Life Questionnaire (PedsQL) in its generic version and the diabetes module. Results: The quality of life assessed by the adolescent with DM1 using Quality of Life Scales is significantly and inversely associated with HbAlc values. This association includes a significant correlation between the total generic quality of life scores and HbAlc but does not have the same impact on specific scores. Conclusions: Metabolic control appears to be the cornerstone that influences the impact on the bidirectional relationship between DM1 and quality of life, however, there is no absolute consensus on the types of factors and degrees that would influence metabolic control.
\end{abstract}

\section{Keywords:}

Adolescent;

Type 1 Diabetes

Mellitus;

Quality of life;

Health Impact

Assessment;

Glycated Hemoglobin

A1c

\section{Introducción}

La diabetes mellitus tipo 1 (DM1) es la enfermedad crónica no transmisible más frecuente en niños y adolescentes, tiene una prevalencia mundial que oscila entre 0,8 y 4,6/1.000 habitantes, siendo el grupo de mayor incidencia el de 10 a 14 años ${ }^{1}$. Estas estimaciones, según la International Diabetes Federation, están aumentando cada año ${ }^{2}$. En este contexto, Chile es el único país latinoamericano que tiene un estudio de incidencia de toda la población del sistema de salud público, lo que corresponde al $80 \%$ de la población general. Los datos extraídos de la notificación obligatoria de la DM1 del programa Garantías Explícitas en Salud (GES) evidencian que la incidencia media anual ha aumentado significativamente desde un 10,2 en 2006 a un 13,8 en 2014, teniendo la mayor tasa el grupo de 10 a 14 años $(16,8 / 100.000)^{3}$.

Esta patología al ser de carácter crónico y requerir de un estricto control metabólico afecta las diversas etapas del ciclo vital de las personas que la padecen ${ }^{4}$. Especialmente afectada se ve la etapa de la adolescencia, la que involucra una fase de transición entre la niñez y la adultez y que "se caracteriza por profundas transformaciones biológicas, psicológicas y sociales, muchas de ellas generadoras de crisis, conflictos y contradicciones" ${ }^{\prime \prime}$. Ambos aspectos, patología y cambios biopsicosociales de estos individuos, pueden repercutir en la calidad de vida de ellos, la cual ha sido definida por la Organización Mundial de la Salud (OMS) como "la percepción del individuo de su posición en la vida en el contexto de la cultura y los sistemas de valores en los que vive y en relación con sus objetivos, expectativas, estándares y preocupaciones" ${ }^{\text {" }}$. Específicamente, el concepto de calidad de vida relacionado con el ámbito de la salud se define "como el nivel de bienestar derivado de la evaluación que la persona realiza de diversos dominios de su vida, considerando el impacto que en estos tiene su estado de salud"7.

De acuerdo a lo expuesto, el objetivo de este estudio fue identificar evidencias científicas sobre el impacto de la diabetes mellitus tipo 1 en la calidad de vida de los adolescentes que padecen esta enfermedad.

\section{Método}

Se realizó una revisión sistemática de la literatura relacionada con el tema de DM1 y adolescentes, de acuerdo a las etapas recomendadas por la Colaboración Cochrane, que son: I) formulación de la pregunta de investigación; II) establecimiento de los criterios de inclusión y exclusión; III) localización de los artículos; IV) aplicación de criterios de calidad metodológica; V) recolección de datos, análisis y VI) presentación de los resultados ${ }^{8,9}$.

Esta revisión sistemática fue guiada por la siguiente pregunta de investigación: ¿qué evidencias científicas existen sobre el impacto de la diabetes mellitus tipo 1 en la calidad de vida de los adolescentes portadores de esta enfermedad? Esta pregunta se sustenta en la estrategia PICoR, la que refiere a: Paciente - adolescente portador de diabetes mellitus tipo 1; Intervención factores o conductas que intervienen en la DM1 y que se relacionan con la calidad de vida de los adolescentes; Comparación - no aplica en esta revisión; Resultados -evaluación del impacto de la DM1 en la salud del adolescente medido por las escalas de calidad de vida. 
Para esta estrategia de búsqueda se utilizaron las bases de datos Medical Literature Analysis and Retrieval System online (MEDLINE), Cumulative Index to Nursing and Allied Health Literature (CINAHL), Literatura Latino-Americana e do Caribe em Ciencias da Saúde (LILACS) y Scientific Electronic Library Online (SciELO) revisadas en el mes de diciembre de 2019.

Se utilizaron descriptores seleccionados en inglés del Medical Subject Headings (MeSH), en español y portugués de los Descriptores en Ciencias de la Salud (DeCS), junto a los operadores booleanos (AND y OR). Se utilizó la siguiente estrategia de búsqueda en MedLine y luego se adaptó a las otras bases de datos: Search $\left(\left(\left(\left(\right.\right.\right.\right.$ Adolescent $\left.^{\star}\right)$ OR Teen $\left.\left.{ }^{\star}\right)\right)$ AND (((Diabetes Mellitus, Type1) OR Diabetes, type 1) OR Type 1 diabetes $))$ AND (((Quality of life) OR Health related quality of life) OR Life quality) $)$ AND $(((()((($ Health impact assessment) OR Health impact) OR Impact assessment, health) OR Diabetes Impact Measurement Scales) OR PedsQL) OR Glycated Hemoglobin A1c) OR Glycosylated Hemoglobin A1c) OR HbA1c) OR Glycemic Control).

Luego a esta búsqueda electrónica se aplicaron los criterios de inclusión y exclusión. Los criterios de inclusión fueron: artículos de investigación, revisiones sistémicas y metaanálisis publicadas en el periodo comprendido entre 2010 y 2019 en español, inglés y portugués. Entre las características particulares del sujeto en estudio se incluyeron: adolescentes (10 a 18 años) con diabetes mellitus tipo 1 . Los criterios de exclusión fueron: revisiones narrativas, guías clínicas, cartas al editor, artículos de opinión, relatos de experiencia, casos clínicos, libros, presentaciones en congresos, estudios en los que no se apreciaba con claridad el método de medición de la variable en estudio y artículos que no respondieran a la pregunta de investigación. Entre las características particulares, se excluyeron a los adolescentes que padecían DM tipo 2 u otras enfermedades crónicas y a los diabéticos institucionalizados; además de los que tenían otras enfermedades asociadas a la DM1.

Una vez identificados los artículos en las bases de datos, se procedió a la eliminación de los registros duplicados mediante el software EndNoteBasic, Thomson Reuters, USA. A continuación, para refinar más la estrategia de búsqueda, se procedió a ejecutar 2 fases de eliminación de registros; la primera, mediante la lectura de títulos y resúmenes, y la segunda, a través de la lectura a texto completo. A este número de artículos seleccionados a texto completo, se aplicaron los criterios de evaluación de calidad metodológica de los estudios, mediante la escala AMSTAR (Assessment of Multiple Systematic Reviews) para las revisiones sistemáticas y para los estudios observacionales, la lista Strenghening the Reporting of Observational Studies in Epidemiology (STROBE) $)^{10,11}$. Los artículos que obtuvieron una puntuación inferior al 80\% fueron eliminados, obteniéndose en esta última fase los artículos incluidos para revisión. Todo este proceso de identificación, selección y evaluación de la elegibilidad de los artículos se realizó mediante la utilización del protocolo Preferred Reporting Items for Systematic Reviews and Meta-Analyse (PRISMA) ${ }^{12}$.

El análisis de los artículos seleccionados para revisión fue realizado por 3 autores de forma independiente y las divergencias fueron resueltas a través de un consenso, después de una nueva revisión del o los artículos en discordancia. Este análisis fue realizado mediante una revisión descriptiva y analítica de los resultados encontrados en la lectura de los artículos a texto completo. Los resultados fueron presentados y evaluados en cuanto a su aplicabilidad o respuesta a la pregunta de investigación en un cuadro sinóptico que considera los siguientes aspectos: identificación del artículo, país de realización, año del estudio, tamaño de la muestra, edad de los participantes, tipo de estudio, criterios de inclusión de los participantes del estudio, escalas de evaluación y el aporte a la pregunta de investigación.

Respecto a los aspectos éticos, fue respetada la integridad de los artículos, es decir, no se realizó modificación alguna de los contenidos encontrados.

\section{Resultados}

Fueron identificados un total de 679 estudios al aplicar la estrategia de búsqueda en las bases de datos Medline, LILACS, CINAHL y Scielo. La trayectoria de identificación, selección, elegibilidad e inclusión de los estudios seleccionados se visualiza en la figura 1 .

Los artículos incluidos en el estudio para su análisis son $25^{13-37}$ (tablas 1 y 2) y la mayoría fueron obtenidos desde la base de datos bibliográfica MEDLINE. El inglés fue el idioma predominante $\mathrm{e}^{13-22,24-35} \mathrm{y}$ en el año 2018 fueron publicados la mayor parte de ellos, $7(28 \%)^{30-36}$. La revista académica más utilizada para la publicación de los artículos fue la Pediatric Diabetes ${ }^{15,26,31,33}$. Se encontraron investigaciones desarrolladas en 4 continentes: en Europa, 9 publicaciones ${ }^{14,15,17,19,22,25,29-31}$; en Asia, 4 publicaciones $^{13,18,20,24}$; en África, 1 publicación ${ }^{28}$ y en América, 11 publicaciones ${ }^{16,19,21,23,26,32-37}$; además de una publicación que abarcó los 5 continentes ${ }^{27}$. En esta diversidad de países se encontró que en los Estados Unidos se realizaron la mayoría de los estudios, $5(20 \%)^{16,19,26,33,34}$ y en América del Sur se destacan Brasil ${ }^{21,32,37}$ y Chile Ch,36. $^{23,3}$. También, se encontraron 2 estudios de revisión narrati$\mathrm{va}^{16,36}$ y 1 con metaánalisis ${ }^{23}$; el resto fueron estudios de tipo transversal descriptivo y, en algunos casos, comparativos de 2 o más cohortes ${ }^{14,15,17,20-22,24-37}$. Por último, 
algunos tuvieron características de estudios multicéntricos nacionales ${ }^{17,19,31}$ e internacionales ${ }^{27}$. Estos últimos estudios fueron los que tuvieron una mayor población de estudio, como el estudio TEEN, que fue uno de los de mayor cobertura mundial realizado entre los años 2012 y 2013 en más de 20 países y 219 centros a nivel mundial con 5.887 niños o jóvenes de 8 a 25 años; considerando temáticas como el manejo de la DM1 y el impacto en los parámetros psicosociales de los portadores de esta patología. La hipótesis comprobada en este estudio observacional de corte transversal fue la existencia de una relación lineal entre calidad de vida y HbAlc y que, según sus autores, probablemente se trate de una relación bidireccional; sin embargo, no se puede establecer una relación de causalidad, porque es un estudio de tipo transversal ${ }^{27}$.

Con relación a los 3 estudios nacionales, se tiene un primer estudio multicéntrico realizado en Noruega de base poblacional. En él se evaluó la calidad de vida de 937 niños y adolescentes con DM1con relación al sistema de administración de la insulina. Los resultados no mostraron diferencias significativas en las puntuaciones entre los usuarios de una bomba de insulina y los de tratamiento de inyección múltiple ${ }^{17}$.

El segundo estudio nacional multicéntrico fue observacional longitudinal de 4 años de seguimiento a una muestra de 1.151 adolescentes del proyecto SEARCH for Diabetes in Youth de Estados Unidos. El objetivo era comparar la calidad de vida de una cohorte de DM1 versus DM2. Los resultados muestran que los adolescentes con DM1 obtuvieron puntuaciones más altas de PedsQL (mejor calidad de vida) que se relacionaron con una mayor educación de los padres, una mayor actividad física y valores más bajos de HbAlc en comparación con los adolescentes portadores de DM2 ${ }^{19}$

Finalmente, el tercer estudio multicéntrico fue de diseño correlacional cuantitativo para evaluar la calidad de vida de 229 adolescentes con DM1 desde el punto de vista principalemente conductual, obteniendo que se puede observar una asociación significativa entre la calidad de vida y la resiliencia ${ }^{31}$.

Con respecto a las características particulares de los sujetos de estudio, la edad de los participantes varió desde los 2 a los 25 años $^{30,33}$ y el promedio de edad se sitúa en un rango que va desde los 10,8 años $^{34}$ a los 16,3 años $^{30,33}$, en los estudios que tenían estos datos; sin embargo, hay 3 estudios en los que fueron incluidos niños junto a adolescentes ${ }^{13,14,22}$. La población de estudio estuvo compuesta por 11.720 participantes; la menor muestra estuvo compuesta por 30 adolescentes, que formaban parte de un campamento de diabetes ${ }^{24}$ y la mayor por 2.846 adolescentes ${ }^{27}$ del estudio TEENs que involucro a 20 países. El criterio de inclusión más común fue que para participar del estudio los adoles-

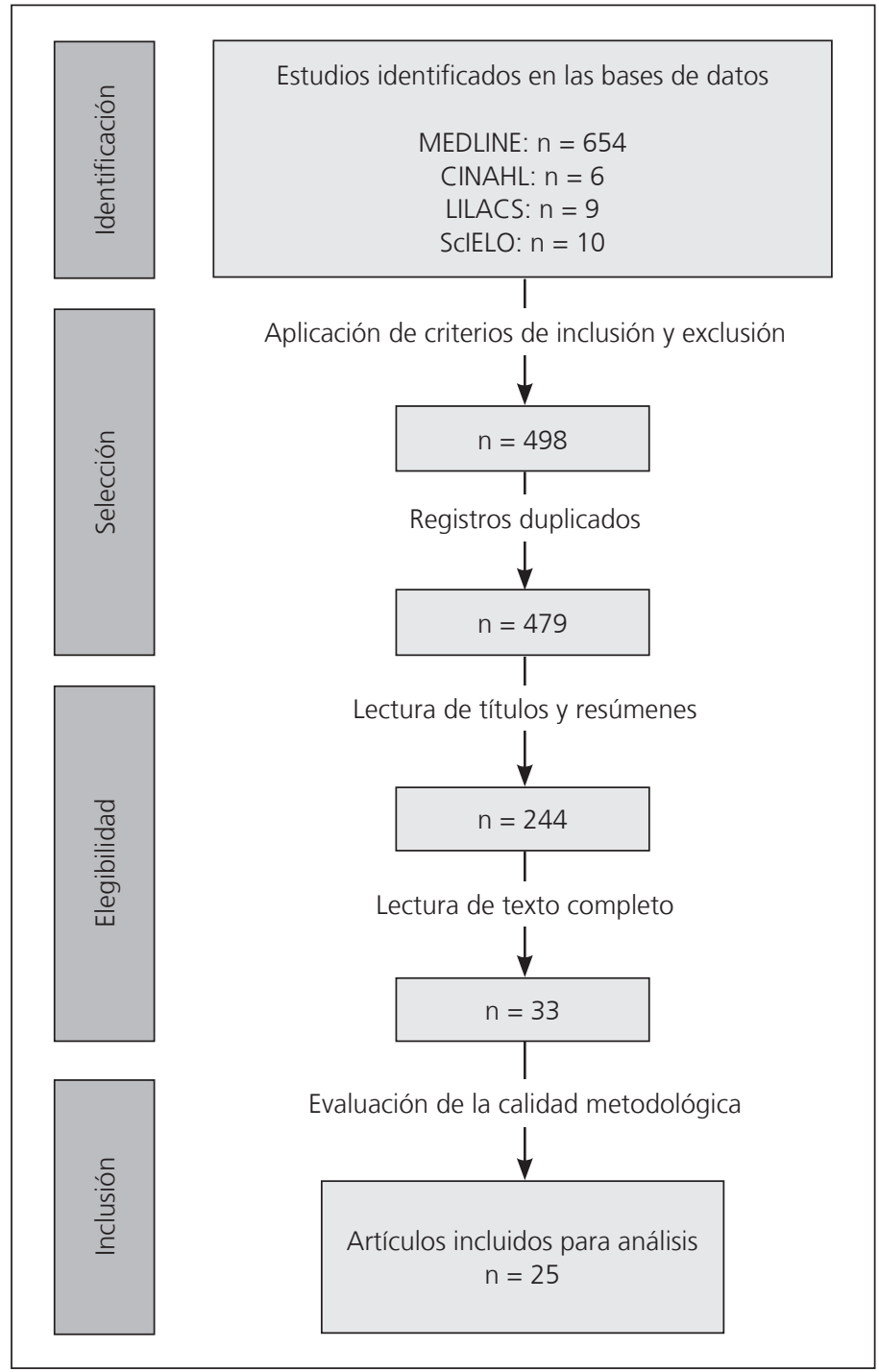

Figura 1. Flujograma de identificación, selección, elegibilidad e inclusión de los estudios. Santiago, Chile, 2020.

centes deberían padecer de DM1como mínimo 1 año desde el diagnóstico ${ }^{18,20,21,23,27-29,31,32,34}$. El instrumento más utilizado, relativo a las mediciones de la calidad de vida, fue el Cuestionario de Calidad de Vida Pediátrica (PedsQL) ${ }^{13,14,18-20,22-25,27,29,31,33,34}$. PedsQL 3.2 Diabetes Module es un instrumento de medición que se utiliza para evaluar la calidad de vida de pacientes pediátricos (2 a 18 años) con DM. Consta de cinco dimensiones que miden los síntomas de la diabetes (15 ítems), barreras de tratamiento (5 ítems), adherencia al tratamiento (6 ítems), preocupación (3 ítems) y comunicación ( 4 ítems). Tiene una excelente validez de constructo y confiabilidad que le confiere la capacidad de evaluar los síntomas y el manejo de la diabetes mellitus en función del impacto sobre la vida cotidiana del paciente. Además, estandariza los resultados en una investigación científica ${ }^{45}$. 


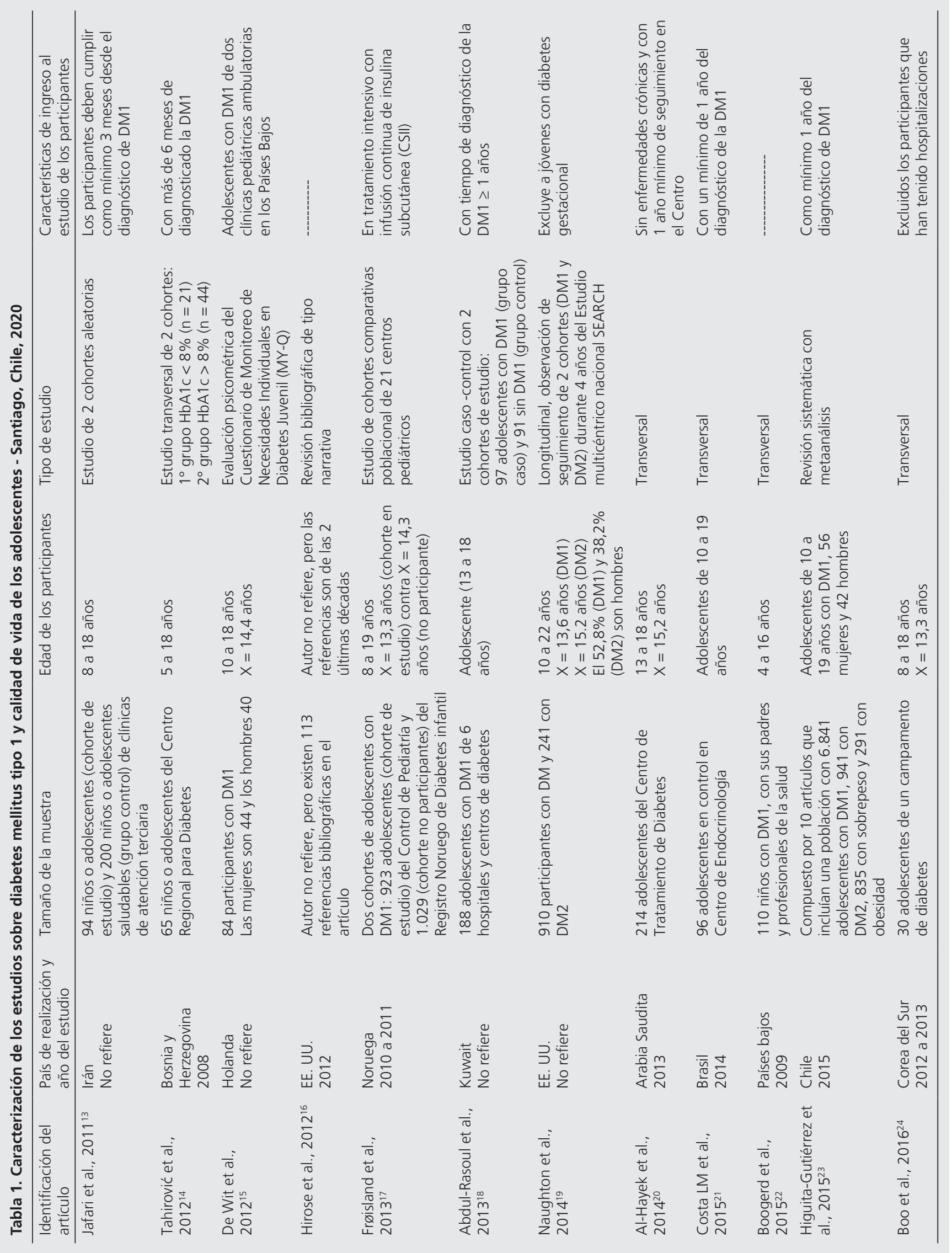




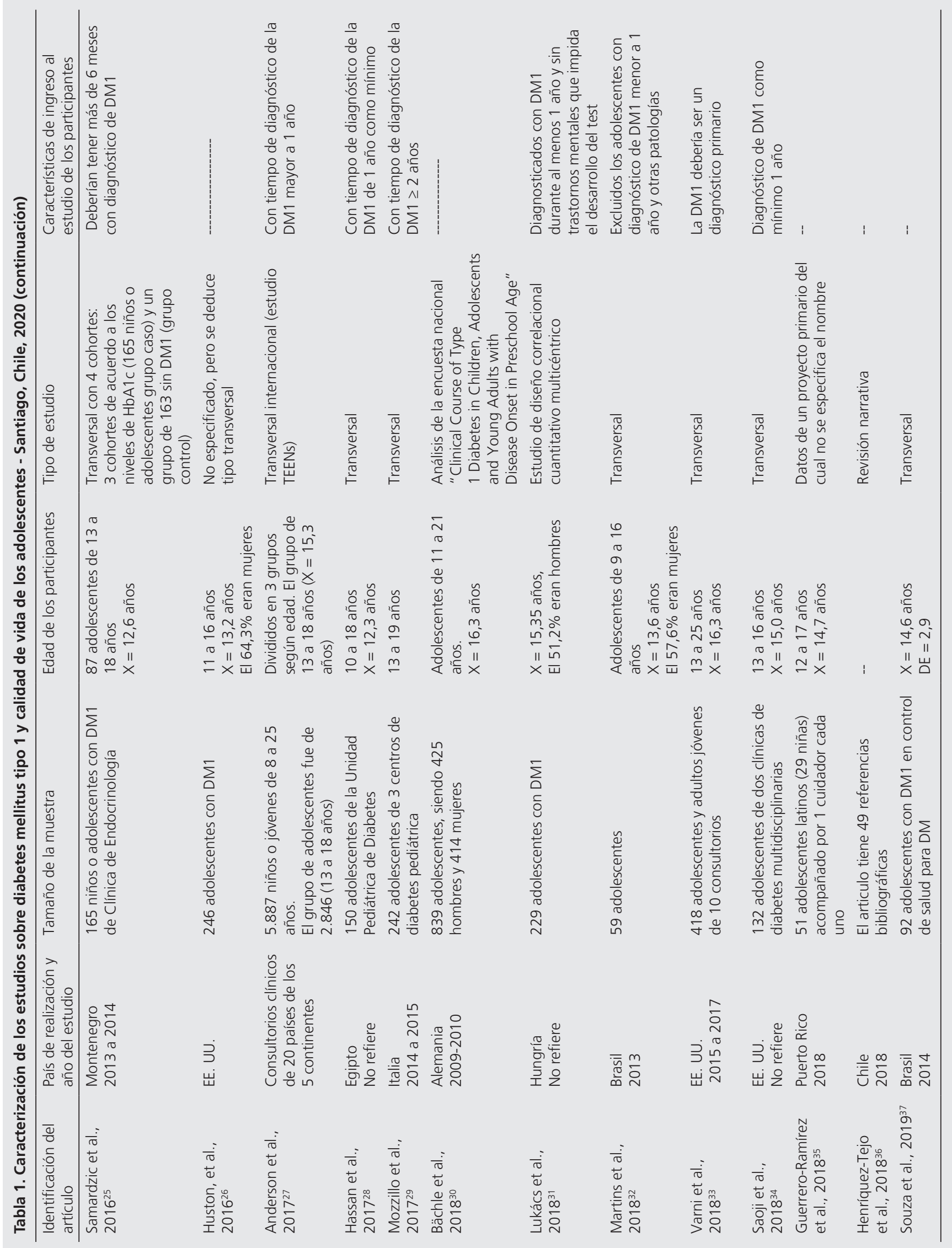




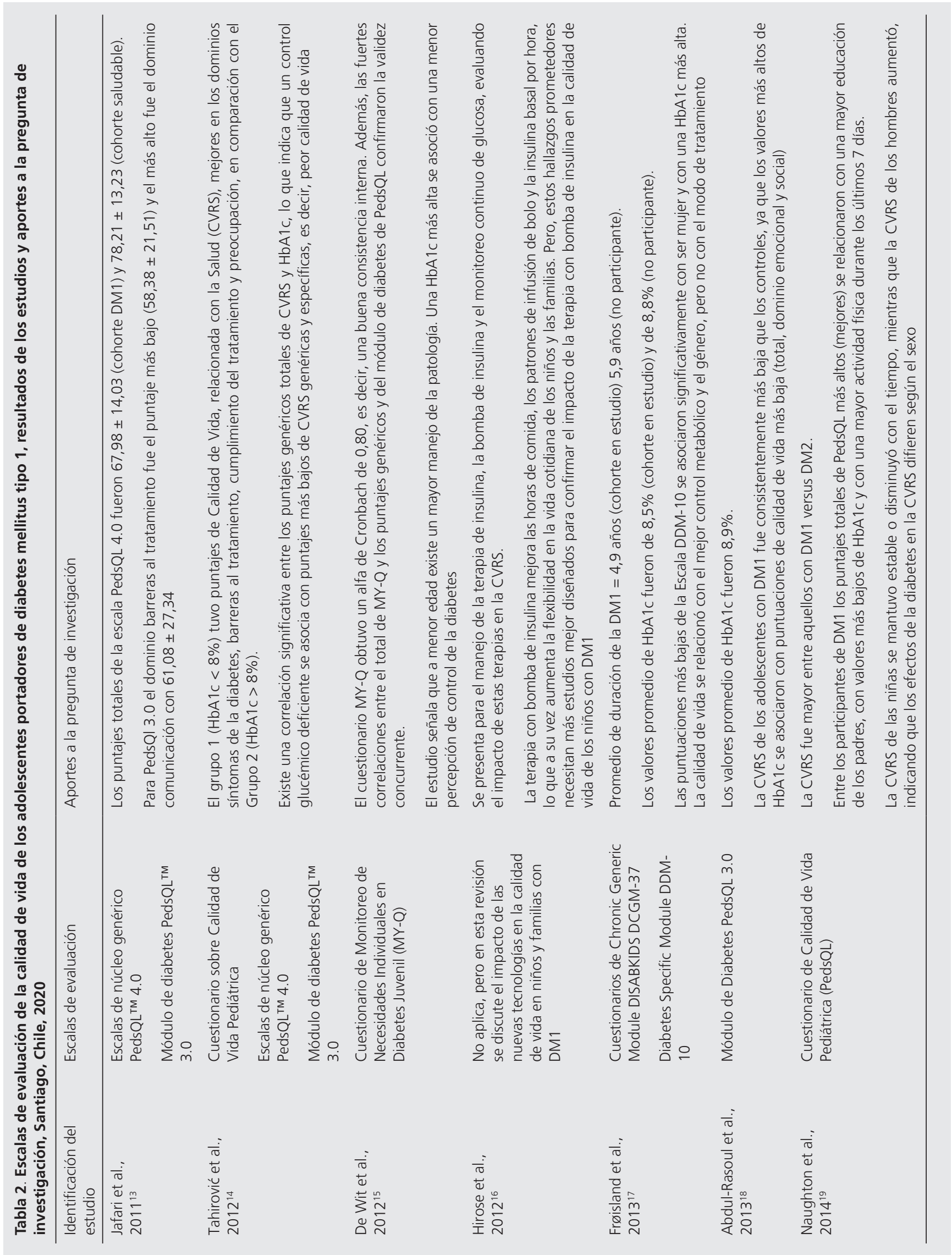




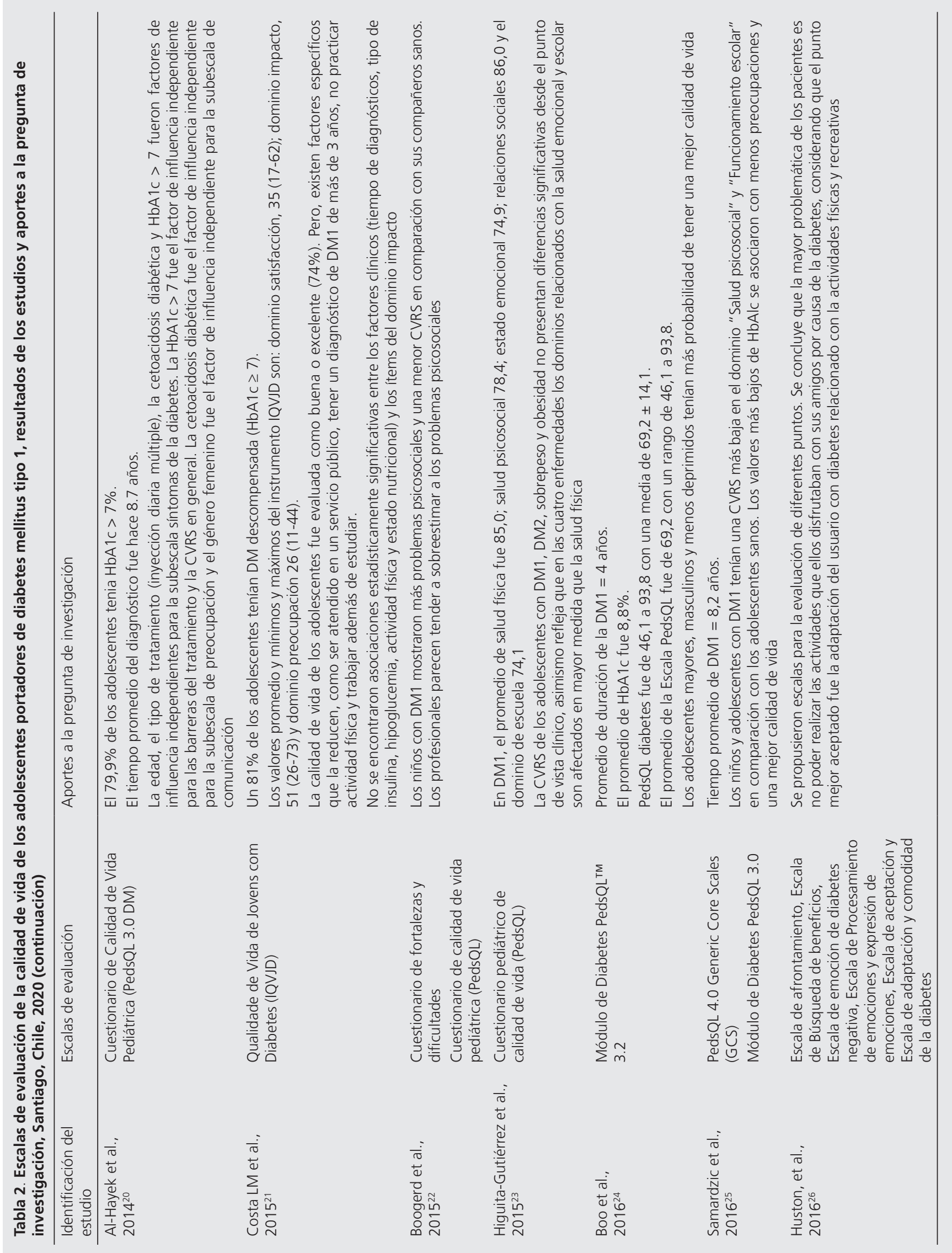




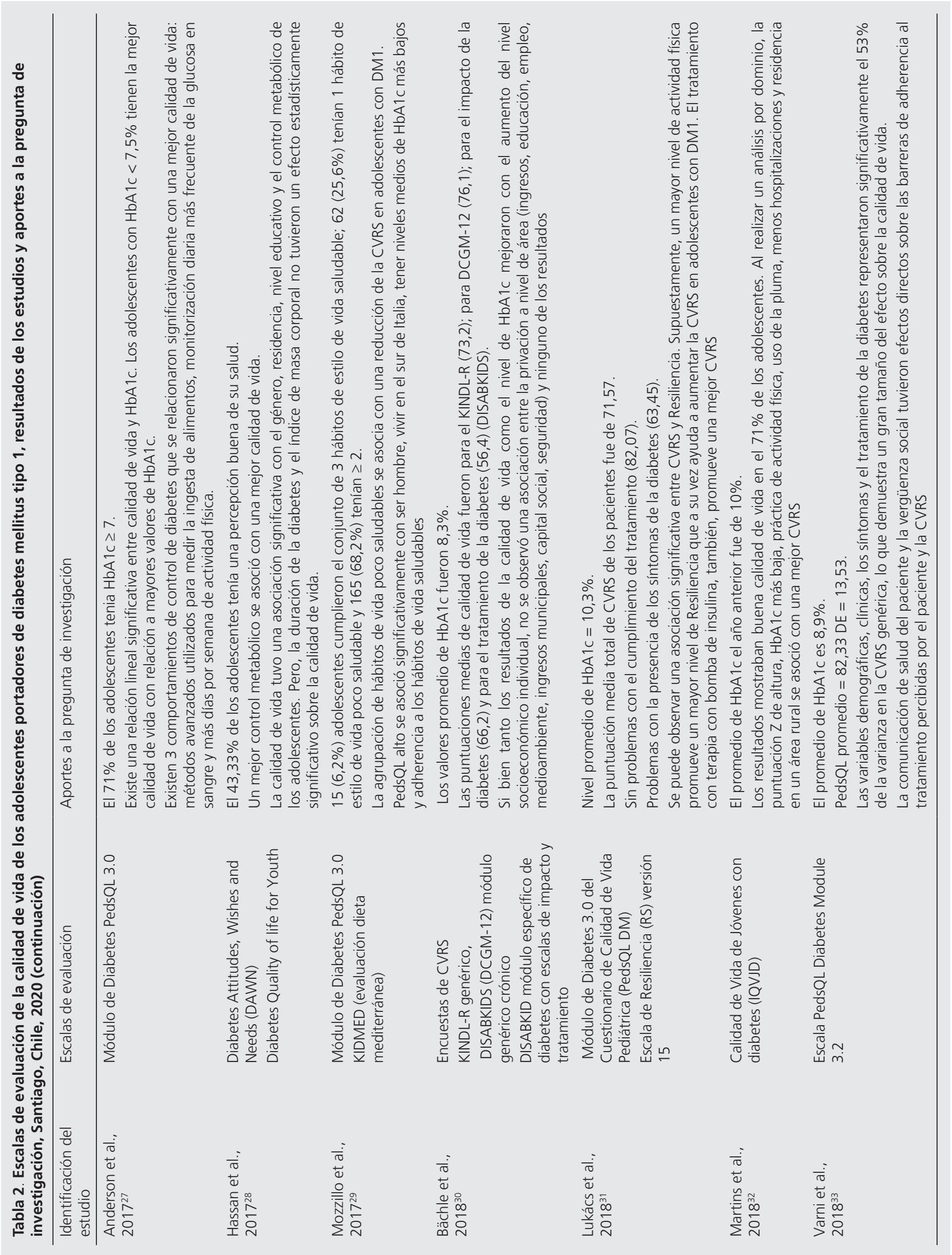




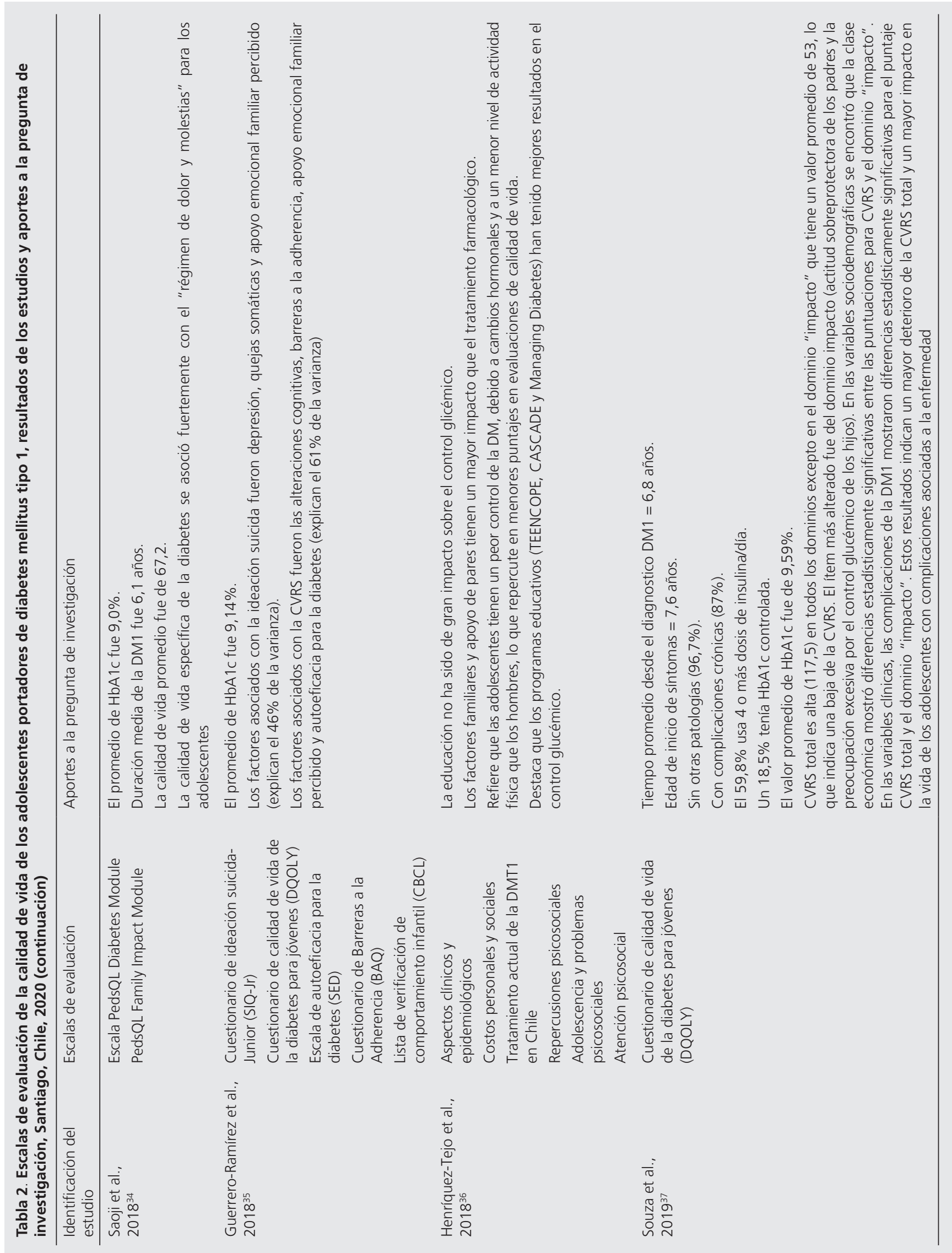




\section{Discusión}

Las evidencias científicas analizadas permiten comprobar que la DM1 está presente en todos los continentes, lo que se constata en los estudios realizados en adolescentes con DM1 en una diversidad de países ${ }^{17-37}$. El estudio internacional TEENs que abarcó una muestra global de 2.846 adolescentes de los 5 continentes, tuvo como objetivo caracterizar la calidad de vida relacionada con la DM1, utilizó un análisis de regresión lineal multivariante y concluyó que la calidad de vida estaba significativamente relacionada con $\mathrm{HbAlc}$, es decir, cuanto más bajo son los valores de $\mathrm{HbAlc}$, mejor es la calidad de vida relacionada con la salud $(\mathrm{CVRS})^{27}$. En este estudio el $71 \%$ de los adolescentes tenía valores $\mathrm{HbAlc} \geq 7$, similar a lo obtenido en otros 12 estudios de esta revisión que arrojan valores promedio de $7,1 \%{ }^{27}$ a $10,3 \%$ de HbAlc ${ }^{31}$. Estos porcentajes se encuentran lejos del punto de corte estipulado por la American Diabetes Association (ADA) e International Society for Pediatric and Adolescent Diabetes (ISPAD), la que establece valores de HbAlc $\leq 7 \%$ como meta ideal para los pacientes diabéticos en tratamiento $^{38,46}$. Sin embargo, varias cohortes de estos estudios están por encima de estos valores de HbAlc, por ende, estos adolescentes presentan una descompensación metabólica de tipo moderada ${ }^{18,33-35,37} \mathrm{y}$, en otros casos, severa $^{31,32}$. Vinculado a este nivel de grado de compromiso metabólico, se verifica la alta prevalencia de descompensación metabólica, como lo demuestra el estudio retrospectivo de 4,5 años de seguimiento realizado en Chile con niños y adolescentes que llevaban un estricto manejo nutricional y un esquema de insulinoterapia, el cual evidenció que solo el $20 \%$ de los casos tenía un control metabólico según normas $\mathrm{ADA}^{47}$. Es por ello que, debido a la relevancia que presenta este análisis sanguíneo definido como "estándar de oro" para monitorizar el control metabólico, es incluido en la evaluación de los aspectos clínicos de la calidad de vida relacionada con la salud (CVRS $)^{38}$.

Otros aspectos que acompañan la evaluación de la calidad de vida son los psicosociales del adolescente que padece DM1. Según Riaño, el adolescente con DM1 se ve afectado en todos los ámbitos de su vida $y$, en especial, en la esfera psicológica ${ }^{39}$. Según Barroso et al., la condición más compleja en estos adolescentes enfermos crónicos es la aceptación y la adaptación a esta situación patológica ${ }^{2,5,40}$. Es tal la importancia de estas alteraciones psicológicas, que su manejo está contemplado en las recomendaciones de las guías clínicas ISPAD Cuidados Psicológicos ${ }^{48}$. Por lo tanto, vivir con DM1 es un desafío para el adolescente.

Para evaluar los aspectos anteriores, existen los Cuestionarios de Calidad de Vida Pediátrica (PedsQL) en versión genérica y módulo diabetes que evalúan la calidad de vida relacionada con la salud (CVRS), los que son ampliamente usados y han sido validados en varios idiomas ${ }^{16,41,42}$. Ambas escalas van de 0 a 100 y las puntuaciones más altas indican mejor calidad de vida o menos síntomas relacionados con la diabetes ${ }^{42,43}$.

En esta revisión de la bibliográfica los cuestionarios PedsQL genérico o específico fueron utilizados en 14 de 25 estudios ${ }^{13,14,18-20,22-25,27,29,31,33,34}$, de los cuales 9 tienen un diseño de investigación de tipo transversal $^{20,22,24,25,27,29,31,33,34}$ y 5 de tipo caso-control ${ }^{13,14,17,18,25}$.

$\mathrm{Al}$ realizar mediciones con estas escalas, se identificó que a menor edad había un mayor manejo de la patología ${ }^{15} \mathrm{y}$ al hacer una comparación por género, esta se asoció significativamente el ser mujer con puntuaciones más bajas, es decir, peor calidad de vida ${ }^{17,19,24,29,36}$.

Los dominios emocional y social de esta Escala PedsQL genérica cuando se asociaron con mayores niveles de $\mathrm{HbAlc}$ tuvieron los puntajes más bajos, como sucedió en los estudios caso-control, en los cuales los puntajes menores en los dominios emocional, social y escolar de la Escala PedsQL correspondían a las cohortes diabéticas al compararlos con cohortes saludables ${ }^{13,18,22,25}$. Autores como Higuita-Gutiérrez et al., reafirman esto en su revisión sistemática y meta análisis, cuando refieren que los dominios salud emocional y escolar son afectados mucho más que la salud física $^{23}$. Este es un hallazgo importante, ya que un estado emocional asociado a niveles más altos de estrés genera mayores niveles de cortisol que interfieren con el metabolismo de la insulina y con ello en el control metabólico ${ }^{23}$. Además, estos aspectos psicológicos se ven relacionados con la salud física, al verificar que una HbA1c más alta se asoció con una menor percepción de control de la diabetes ${ }^{15}$. Estos resultados conducen a los estudios que reafirman que los altos niveles de este indicador (HbAlc) se asocian con un puntaje de CVRS más bajos (peor calidad de vida) 14,17,18,20,25,27-29,32. Estos estudios mostraron que esta patología requiere, por parte del adolescente, un autocontrol diario que es la piedra angular del cuidado de esta patología.

La Escala PedsQL diabetes pretende evaluar los dominios específicos de esta patología que, en algunos estudios, muestra que los mejores puntajes en dominios síntomas de la diabetes, barreras al tratamiento, adhesión al tratamiento y preocupación, se relacionan con un mejor control glucémico ${ }^{14,25,33,35}$. Estos resultados han sido reafirmados en un estudio caso-control en el que se compararon 2 grupos de adolescentes con DM1 que tenían diferentes niveles promedios de control metabólico $(7,3 \%$ \& 8,8\% de HbAlc) y se ratificó la tendencia de los resultados anteriores, es decir, los adolescentes con un mejor control metabólico tienen mejores puntajes en las dimensiones de la Escala PedsQL diabetes, excepto en la dimensión "comunicación” ${ }^{14}$. Sin embargo, autores como Frøisland et al., no 
obtuvieron los mismos hallazgos en su investigación, pues encontraron que la calidad de vida no se relacionó con la adhesión al tratamiento ${ }^{17}$.

Al utilizar otras escalas, como el cuestionario de calidad de vida de la diabetes para jóvenes (DQOLY), el ítem más alterado fue el dominio impacto (actitud sobreprotectora de los padres y su preocupación excesiva por el control glucémico de los hijos) ${ }^{37}$. Un resultado diferente se obtuvo con la misma escala anterior, en la cual entre el dominio impacto y los factores clínicos (como tipo de insulina, hipoglucemia, actividad física y estado nutricional) no se encontró una asociación estadísticamente significativa ${ }^{21}$. Otros estudios, como el de Souza et al., encontraron que las variables clínicas mostraron diferencias estadísticamente significativas para el puntaje CVRS total y el dominio impacto ${ }^{37}$. En esta escala los resultados indicaron un mayor deterioro de la CVRS total y que el mayor impacto en la vida de los adolescentes son las complicaciones asociadas a la enfermedad derivado del mal manejo del control metabólico ${ }^{37}$.

Relacionado con los postulados anteriores, Anderson et al., refieren que uno de los 3 comportamientos más relevantes para el control de la diabetes, que se relacionó significativamente con una mejor calidad de vida, es la monitorización diaria de la glucosa en sangre ${ }^{27}$, por lo tanto, el tratamiento con una terapia con bomba de insulina ayudaría a este hecho. Esta idea es también compartida por Lukács et al., quien comprobó que la bomba de insulina promueve una mejor $\mathrm{CVRS}^{31}$. Pero este parecer no es ratificado por Hirose et al., quienes presentaron, mediante una revisión narrativa, que los estudios con esta tecnología de terapia de insulina tienen diseños metodológicos deficientes y que por ello sus evaluaciones son inconsistentes con la calidad de vida, por lo tanto, el autor plantea que limitan el poder afirmar que existe una fuerte asociación entre los beneficios de la terapia con bomba de insulina y la calidad de vida ${ }^{16}$.

Con respecto a otros comportamientos, como los estilos de vida, se puede observar que los puntajes totales de PedsQL más altos se relacionaron con valores más bajos de HbAlc y con una mayor actividad físi$\mathrm{ca}^{19,27,29,31,32,36}$.

Esta afirmación también se presentó al comparar un grupo de adolescentes con DM1 con otro con DM2, lo que arrojó como resultado que una mejor CVRS en los DM1 se relaciona con una mayor actividad física, con una mayor educación de los padres y con valores más bajos de HbA1 $\mathrm{c}^{19}$; sin embargo, otros trabajos refieren que tanto la calidad de vida como el nivel de HbAlc mejoraron con el aumento del nivel socioeconómico individual, pero no se observó una asociación con la disminución de los ingresos, la educación, el empleo, el capital social y la seguridad, entre otros aspectos ${ }^{30}$. Otro aspecto importante en el ciclo de vida para estos adolescentes con DM1 es emigrar desde un sistema de atención pediátrica a uno de atención para adultos. Con el objetivo de conocer este proceso, se realizó un interesante estudio en Chile con el fin de evaluar la efectividad de un "programa de transición" con 2 grupos de estudios; uno con intervenciones de atención integral del equipo de salud y otro sin ellas. Los resultados mostraron que los adolescentes con dicha intervención tenían mejores indicadores de adherencia para mantener o mejorar los niveles de HbA1c (60\% frente a $30 \%$ ), sin embargo, esto no fue significativo ${ }^{49}$.

Estos estudios fueron publicados en revistas científicas especificas del tema como Pediatric diabetes $^{15,26,31,33}$, Journal of Pediatric Endocrinology and Metabolism ${ }^{25,28}$, Current Diabetes Reports ${ }^{16}$ y Diabetes care ${ }^{27}$. La revisión y análisis realizado, evidencia la existencia de una gran preocupación de la comunidad científica para contribuir a enfrentar esta dolencia, lo que representa un respaldo de la ciencia, que contribuye a dar respuesta a la pregunta de investigación planteada en este trabajo.

En conclusión, la calidad de vida medida por los propios adolescentes diabéticos está significativamente asociada a los valores de HbAlc, es decir, cuanto más alto es el nivel de este indicador más bajo es el puntaje de CVRS (peor calidad de vida). Esta vinculación se extiende a una correlación significativa entre los puntajes genéricos totales de CVRS y HbA1c, pero no tiene la misma repercusión en los puntajes CVRS específicos, por la existencia de controversias en algunas dimensiones de esta escala.

Así, el control metabólico se establece como la piedra angular que incide en el impacto en la relación DM1 y calidad de vida; vinculación que se vislumbra como bidireccional, aunque no se evidencia un consenso absoluto sobre los tipos de factores y los grados que influirían en el control metabólico.

\section{Conflicto de intereses}

Los autores declaran no tener conflicto de intereses. 


\section{Referencias}

1. Forga L. Epidemiología en la diabetes tipo 1: ayudando a encajar las piezas del puzzle. Endocrinol Nutr. 2015;62(4):14951. http://dx.doi.org/10.1016/j. endonu.2015.02.002.

2. International Diabetes Federation. IDF Diabetes atlas. Ninth edition 2019. Disponible: www.diabetesatlas.org.

3. Garfias CP, Borzutzky A, Ugarte MF, García HJ, Phingsthorn M, García HG. Mandatory notifications of type 1 diabetes incident cases in Chilean children, 2006 to 2014: A population-based study. Pediatr Diabetes. 2020;21(1):48-52. doi:10.1111/ pedi.12937.

4. Henríquez-Tejo R, Cartes-Velásquez R. Impacto psicosocial de la diabetes mellitus tipo 1 en niños, adolescentes y sus familias. Revisión de la literatura. Rev Chil Pediatr. 2018;89(3):391-8. doi: 10.4067/ S0370-41062018005000507.

5. Pineda S, Aliño M. El concepto de la adolescencia. In: Manual de prácticas clínicas para la atención integral a la salud en la adolescencia. Ministerio de Salud Pública MINSAP. La Habana, Cuba, 2a edición, 2002. P. 15-23.

6. Urzúa M, Caqueo-Urízar A. Calidad de vida: Una revisión teórica del concepto. Ter Psicol. 2012;30(1):6171. http://dx.doi.org/10.4067/S071848082012000100006.

7. Urzúa A. Calidad de vida relacionada con la salud: Elementos conceptuales. Rev Med Chile 2010;138:358-65. http://dx.doi. org/10.4067/S0034-98872010000300017.

8. Barbosa FT, Lira AB, Oliveira Neto $\mathrm{OB}$, et al. [Tutorial for performing systematic review and meta-analysis with interventional anesthesia studies]. Rev Bras Anestesiol. 2019;69(3):299-306. doi:10.1016/j.bjan.2018.11.007.

9. Higgins JPT, Green S (editors). Cochrane Handbook for Systematic Reviews of Interventions Version 5.1.0 [updated March 2011]. The Cochrane Collaboration, 2011. Disponible: www. cochrane-handbook.org.

10. Assessment the methodological quality of systematic reviews (AMSTAR). The development of AMSTAR. Disponible: https://amstar.ca/Amstar Checklist.php.

11. STROBE statement. STrengthening the Reporting of OBservational studies in Epidemiology. Disponible: https:// www.strobe-statement.org/index. php?id=strobe-home.

12. Moher D, Shamseer L, Clarke M, Ghersi D, Liberati A, Petticrew M, et PRISMA-P Group. Preferred reporting items for systematic review and meta-analysis protocols (PRISMA-P) 2015 statement. Syst Rev. 2015;4:1. doi: 10.1186/2046-4053-4-1.
13. Jafari P, Forouzandeh E, Bagheri Z, Karamizadeh Z, Shalileh K. Health related quality of life of Iranian children with type 1 diabetes: reliability and validity of the Persian version of the PedsQL ${ }^{\mathrm{TM}}$ Generic Core Scales and Diabetes Module. Health Qual Life Outcomes. 2011;9:104. doi: 10.1186/1477-7525-9-104.

14. Tahirović $\mathrm{H}$, Toromanović A, Tahirović E, Begić H, Varni JW. Health-related quality of life and metabolic control in children with type 1 diabetes mellitus in Bosnia and Herzegovina. Coll Antropol. 2012;36(1):117-21.

15. De Wit M, Winterdijk P, Aanstoot HJ, et al. Assessing diabetes-related quality of life of youth with type 1 diabetes in routine clinical care: the MIND Youth Questionnaire (MY-Q). Pediatr Diabetes. 2012;13(8):638-46. doi: 10.1111/j.13995448.2012.00872.x.

16. Hirose M, Beverly EA, Weinger K. Quality of life and technology: impact on children and families with diabetes. Curr Diab Rep. 2012;12(6):711-20. doi: 10.1007/s11892012-0313-4.

17. Frøisland DH, Graue M, Markestad T, Skrivarhaug T, Wentzel-Larsen T, Dahl-Jørgensen K. Health-related quality of life among Norwegian children and adolescents with type 1 diabetes on intensive insulin treatment: a population-based study. Acta Paediatr. 2013;102(9):889-95. doi: 10.1111/ apa.12312.

18. Abdul-Rasoul M, AlOtaibi F, Abdulla A, Rahme Z, AlShawaf F. Quality of life of children and adolescents with type 1 diabetes in Kuwait. Med Princ Pract. 2013;22(4):379-84. doi: $10.1159 / 000347052$.

19. Naughton MJ, Yi-Frazier JP, Morgan TM, et al. Longitudinal associations between sex, diabetes self-care, and health-related quality of life among youth with type 1 or type 2 diabetes mellitus. J Pediatr. 2014;164(6):1376-83.el. doi: 10.1016/j. jpeds.2014.01.027.

20. Al-Hayek AA, Robert AA, Abbas HM, et al. Assessment of health-related quality of life among adolescents with type 1 diabetes mellitus in Saudi Arabia. Saudi Med J. 2014;35(7):712-7.

21. Costa LM, Vieira SE. Quality of life of adolescentes with type 1 diabetes. Clinics (Sao Paulo). 2015;70(3):173-9. doi: 10.6061/clinics/2015(03)04.

22. Boogerd EA, Damhuis AM, van Alfenvan der Velden J, et al. Assessment of psychosocial problems in children with type 1 diabetes and their families: the added value of using standardised questionnaires in addition to clinical estimations of nurses and paediatricians. J Clin Nurs. 2015;24(15-16):2143-51. doi: 10.1111/jocn.12789.
23. Higuita-Gutiérrez LF, Vargas-Alzate CA, Cardona-Arias JA. Impacto de la diabetes, el sobrepeso y la obesidad en la calidad de vida relacionada con la salud del adolescente: metanálisis. Rev chil nutr. 2015;42(4):383-91. doi:10.4067/S071775182015000400010 .

24. Boo S, Ahn Y, Lee JE, Kang N, Kang H, Sohn M. Diabetes-Specific Quality of Life of Korean Children and Adolescents With Type 1 Diabetes. Int J Nurs Knowl. 2016;27(2):62-9. doi: 10.1111/20473095.12075 .

25. Samardzic M, Tahirovic H, Popovic N, Popovic-Samardzic M. Health-related quality of life in children and adolescents with type 1 diabetes mellitus from Montenegro: relationship to metabolic control. J Pediatr Endocrinol Metab. 2016;29(6):663-8. doi: 10.1515/jpem2015-0420.

26. Huston SA, Blount RL, Heidesch T, Southwood R. Resilience, emotion processing and emotion expression among youth with type 1 diabetes. Pediatr Diabetes. 2016;17(8):623-31. doi: 10.1111/ pedi.12347.

27. Anderson BJ, Laffel LM, Domenger C, et al. Factors associated with diabetesspecific health-related quality of life in youth with type 1 diabetes: The Global TEENs Study. Diabetes Care. 2017;40(8):1002-9. doi: 10.2337/dc161990.

28. Hassan M, Musa N, Abdel Hai R, Fathy A, Ibrahim A. Assessment of health-related quality of life in Egyptian adolescents with type 1 diabetes: DEMPU survey. J Pediatr Endocrinol Metab. 2017;30(3):277-83. doi: 10.1515/jpem-2016-0147.

29. Mozzillo E, Zito E, Maffeis C, et al. Unhealthy lifestyle habits and diabetesspecific health-related quality of life in youths with type 1 diabetes. Acta Diabetol. 2017;54(12):1073-80. doi: 10.1007/s00592-017-1051-5.

30. Bächle C, Peneva A, Maier W, et al. Association of individual and area-level socioeconomic conditions with quality of life and glycaemic control in 11- to 21-year-old adolescents with early-onset type 1 diabetes: a cross-sectional study. Qual Life Res. 2018;27(12):3131-6. doi: 10.1007/s11136-018-1949-6.

31. Lukács A, Mayer K, Sasvári P, Barkai L. Health-related quality of life of adolescents with type 1 diabetes in the context of resilience. Pediatr Diabetes. 2018;19(8):1481-6. doi: 10.1111/ pedi.12769.

32. Martins KAKF, Mascarenhas LPG, Morandini M, et al. Health-related quality of life in a cohort of youths with type 1 diabetes. Rev Assoc Med Bras (1992). 2018;64(11):1038-44. doi: 10.1590/18069282.64.11.1038. 
33. Varni JW, Delamater AM, Hood KK, et al. Diabetes management mediating effects between diabetes symptoms and health-related quality of life in adolescents and young adults with type 1 diabetes. Pediatr Diabetes. 2018;19(7):1322-30. doi: $10.1111 /$ pedi.12713.

34. Saoji N, Palta M, Young HN, Moreno MA, Rajamanickam V, Cox ED. The relationship of Type 1 diabetes selfmanagement barriers to child and parent quality of life: a US cross-sectional study. Diabet Med. 2018;35(11):1523-30. doi: 10.1111/dme.13760.

35. Guerrero-Ramírez G, Cumba-Avilés E. Factors Associated with Suicidal Ideation and Quality of Life in Adolescents from Puerto Rico with Type 1 Diabetes. P R Health Sci J. 2018;37(1):19-21.

36. Henríquez-Tejo R, Cartes-Velásquez R. Impacto psicosocial de la diabetes mellitus tipo 1 en niños, adolescentes y sus familias. Revisión de la literatura. Rev. chil. pediatr. 2018;89(3):391-8. http://dx.doi.org/10.4067/S037041062018005000507.

37. Souza MA, Freitas RWJF, Lima LS, Santos MA, Zanetti ML, Damasceno MMC. Health-related quality of life of adolescentes with type 1 diabetes melltus. Rev. Latino-Am Enfermagem. 2019;27:e3210. doi: 10.1590/15188345.2961.3210.

38. Campuzano-Maya G, Latorre-Sierra G. La
HbAlc en el diagnóstico y en el manejo de la diabetes. Medicina \& Laboratorio 2010;16:211-41. Disponible: https://www. medigraphic.com/pdfs/medlab/myl-2010/ myl105-6b.pdf.

39. Riaño I. Calidad de vida en enfermedades endocrinológicas. Rev Esp Endocrinol Pediatr. 2017;8 Suppl(1):86-93. doi: 10.3266/RevEspEndocrinolPediatr. pre2017.Apr.395.

40. Barroso A, Castillo G, Benitez N, Leyva A. Repercusión y tratamiento de los aspectos psicosociales de la diabetes mellitus tipo 1 en adolescentes. Rev Cubana Pediatr 2015;87(1):92-101. Disponible: http:// scielo.sld.cu/pdf/ped/v87n1/ped11115.pdf

41. Varni JW. The PedsQL. Measurement model for the pediatric quality of life inventory. Disponible: https://www. pedsql.org/index.html.

42. Varni JW, Burwinkle TM, Jacobs JR, Gottschalk M, Kaufman F, Jones KL. The PedsQL in type 1 and type 2 diabetes: reliability and validity of the Pediatric Quality of Life Inventory Generic Core Scales and type 1 Diabetes Module. Diabetes Care. 2003;26(3):631-7. doi: 10.2337/diacare.26.3.631.

43. Millán M. Cuestionario de calidad de vida específico para la diabetes mellitus (EsDQOL). Aten Primaria 2002;29(8): 517-21.

44. Díaz-Cárdenas C, Wong C, Vargas N. Grado de control metabólico en niños y adolescentes con diabetes mellitus tipo 1 . Rev chil pediatr. 2016;87(1):43-7. http:// dx.doi.org/10.1016/j.rchipe.2015.09.002.

45. Varni JW, Delamater AM, Hood KK, et al. PedsQL 3.2 Diabetes module for children, adolescents, and young adults: reliability and validity in type 1 diabetes. Diabetes Care. 2018;41(10):2064-2071. doi: $10.2337 / \mathrm{dc} 17-2707$.

46. DiMeglio LA, Acerini CL, Codner E, et al. ISPAD Clinical Practice Consensus Guidelines 2018: glycemic control targets and glucose monitoring for children, adolescents, and young adults with diabetes. Pediatr Diabetes 2018;19 Suppl 27:105-14. doi: 10.1111/pedi.12737.

47. Díaz-Cárdenas C, Wong C, Vargas N. Grado de control metabólico en niños y adolescentes con diabetes mellitus tipo 1. Rev chi. pediatr. 2016;87(1):43-7. http:// dx.doi.org/10.1016/j.rchipe.2015.09.002.

48. Delamater AM, de Wit M, McDarby V, et al. ISPAD Clinical Practice Consensus Guidelines 2018: Psychological care of children and adolescents with type 1 diabetes. Pediatr Diabetes. 2018;19 Suppl 27:237-49. doi:10.1111/pedi.12736.

49. Santibáñez G, Rubio C, Toro E, et al. Programa de transición: una intervención para adolescentes con diabetes tipo 1: estudio de casos y controles. Rev chil endocrinol diabetes 2018;11(1):710. Disponible: http://revistasoched. cl/1_2018/2.pdf. 